\title{
Optimizing Train Stopping Patterns for Congestion Management*
}

\author{
Tatsuki Yamauchi ${ }^{1}$, Mizuyo Takamatsu $^{\dagger 2}$, and Shinji Imahori ${ }^{\ddagger 3}$ \\ 1 Department of Information and System Engineering, Chuo University, Tokyo, \\ Japan \\ a13.fshw@g . chuo-u.ac .jp \\ 2 Department of Information and System Engineering, Chuo University, Tokyo, \\ Japan \\ takamatsu@ise.chuo-u.ac.jp \\ 3 Department of Information and System Engineering, Chuo University, Tokyo, \\ Japan \\ imahori@ise.chuo-u.ac.jp
}

\begin{abstract}
In this paper, we optimize train stopping patterns during morning rush hour in Japan. Since trains are extremely crowded, we need to determine stopping patterns based not only on travel time but also on congestion rates of trains. We exploit a Wardrop equilibrium model to compute passenger flows subject to congestion phenomena and present an efficient local search algorithm to optimize stopping patterns which iteratively computes a Wardrop equilibrium. We apply our algorithm to railway lines in Tokyo including Keio Line with six types of trains and succeed in relaxing congestion with a small effect on travel time.
\end{abstract}

1998 ACM Subject Classification G.1.6 Optimization, G.2.2 Graph Theory, G.2.3 Applications

Keywords and phrases Train stopping pattern, Wardrop equilibrium, Congestion management, Local search algorithm, Event-activity network

Digital Object Identifier 10.4230/OASIcs.ATMOS.2017.13

\section{Introduction}

In Japan, about seven million people commute to office, school, or university by train every morning. During morning rush hour, trains are very crowded especially in Tokyo. The congestion rate exceeds $200 \%$ for the most crowded train (see Table 1 for congestion rates).

A straight approach to reduce congestion is to increase the number of trains. However, most railway lines in Tokyo have over 25 services per hour during morning rush hour. Thus, the number of services seems to reach a limit. Railway companies in Japan make an effort to relax congestion by another approach: changing timetables and train stopping patterns [15].

During morning rush hour, passengers make a decision about whether to get on an express train or a local train, based on travel time and congestion rates. Some passengers get on a crowded express train, because an express train runs faster than a local train. Some passengers get on a local train at the expense of travel time to avoid congestion. Others leave home early to avoid the rush hour. We need to compute when and which type of train each passenger gets on in view of congestion of trains.

\footnotetext{
* This work was supported in part by JST CREST, Grant Number JPMJCR14D2, Japan.

$\dagger$ The second author's research is supported in part by JSPS KAKENHI Grant Number 16K16356.

¥ The third author's research is supported in part by JSPS KAKENHI Grant Number 15H02969.
} 
Table 1 Examples of trains with high congestion rate [9].

\begin{tabular}{l|l}
\hline Congestion rate: $100 \%$ & Passengers can take a seat or hold on to a strap. \\
Congestion rate: $150 \%$ & Passengers can open up a newspaper and read it. \\
Congestion rate: $200 \%$ & Passengers touch someone's body. \\
Congestion rate: $250 \%$ & Passengers cannot move at all even if trains are bumpy. \\
\hline
\end{tabular}

A Wardrop equilibrium model is commonly used to describe traffic patterns subject to congestion phenomena in road networks [5]. We can compute the behavior of each passenger on trains by finding a Wardrop equilibrium in the event-activity network, which represents timetables of trains and behavior of passengers. As a cost function in a Wardrop equilibrium model, we use the BPR function (Bureau of Public Roads [2]), because the travel cost is determined not only by travel time but also by congestion rates.

In Japan, Taguchi [14] exploits a Wardrop equilibrium in the event-activity network (called a time-space network in Japan), to analyze precisely commuter traffic flow in Tokyo Metropolitan area. In modeling of passenger flows during morning rush hour in Japan, the validity of the Wardrop equilibrium model with the BPR function is shown in [14] by comparing the computational results to the census data for commuter traffic published by Ministry of Land, Infrastructure, Transport and Tourism.

The aim of this paper is to optimize train stopping patterns during morning rush hour. Given stopping patterns and timetables can be evaluated by a Wardrop equilibrium in the event-activity network. In optimization of stopping patterns, however, it is preferable to avoid computation on the event-activity network. One reason is that computing a Wardrop equilibrium in the event-activity network requires much time, which means even evaluation of stopping patterns is computationally expensive. Another reason is that if we change stopping patterns, we have to construct an event-activity network by solving the timetabling problem.

In order to devise an efficient algorithm, we introduce a train type network, which simplifies the event-activity network. We show that a Wardrop equilibrium in the train type network approximates that in the event-activity network. By using the train type network, we can compute a Wardrop equilibrium in only 0.36 seconds without constructing an event-activity network. We present an efficient local search algorithm which iteratively computes a Wardrop equilibrium in the train type network. Our algorithm is applied to railway lines in Tokyo including Keio Line with six types of trains.

Finally, we compare previous work and our results. To determine train stopping patterns is an important issue which is closely related to line planning and timetabling $[7,13]$. Previous work including $[3,8,16,17]$ tackles the problem of optimizing stopping patterns. A big difference between the previous work and this paper is the level of congestion. Trains in Japan are extremely crowded during morning rush hour. Thus, we need to determine stopping patterns with careful consideration of congestion rates of trains. We exploit a Wardrop equilibrium model to compute passenger flows subject to congestion phenomena.

\section{Wardrop equilibrium}

Wardrop's first principle of route choice is the following [4]:

The journey times on all the routes actually used are equal, and less than which would be experienced by a single vehicle on any unused route.

According to Wardrop's first principle, each passenger chooses his or her route to minimize the route cost. Since Wardrop's first principle describes the spreading of trips over alternative 
routes due to congestion, Wardrop equilibrium models have been used to predict route choices of commuters [5].

Let $\mathcal{G}=(\mathcal{V}, \mathcal{A})$ be a directed graph and $\mathcal{C} \subseteq \mathcal{V} \times \mathcal{V}$ be a set of commodities represented by OD pairs. Given an OD pair $k \in \mathcal{C}$, we denote by $\mathcal{R}_{k}$ the set of routes in $\mathcal{G}$ which connect the origin and the destination. The set of all routes is represented by $\mathcal{R}=\bigcup_{k \in \mathcal{C}} \mathcal{R}_{k}$.

A route flow $h=\left(h_{r}\right)_{r \in \mathcal{R}}$ is called a Wardrop equilibrium if and only if it holds that

$$
\sum_{r \in \mathcal{R}_{k}} h_{r}=d_{k} \quad \text { and } \quad \sum_{a \in r} t_{a}\left(f_{a}\right)=\min _{q \in \mathcal{R}_{k}} \sum_{a \in q} t_{a}\left(f_{a}\right) \text { for all } r \in \mathcal{R}_{k} \text { with } h_{r}>0,
$$

where $d_{k}$ is demand, $t_{a}(\cdot)$ denotes a link cost function, and $f=\left(f_{a}\right)_{a \in \mathcal{A}}$ is an arc flow determined by $f_{a}=\sum_{r \ni a} h_{r}$. The first equation says that a route flow meets the demand, and the second one represents that each passenger travels along a path with the minimum cost.

Beckmann et al. [1] proved that we can find a Wardrop equilibrium by solving the following optimization problem, where $X_{f}$ denotes the set of feasible arc flows:

$$
\min \left\{\sum_{a \in \mathcal{A}} \int_{0}^{f_{a}} t_{a}(z) \mathrm{d} z \mid f \in X_{f}\right\} .
$$

This problem can be solved by the Frank-Wolfe method [6].

As explained in Section 3, the event-activity network represents timetables of trains and behavior of passengers. In order to compute the number of passengers in each train accurately, it is significant to find a Wardrop equilibrium in the event-activity network instead of the railway network.

Similar approach has been developed by Taguchi [14] in Japan, where he deals with commuter traffic flows in Tokyo Metropolitan area. He focused on 1,815 stations and 7,486 trains (on 128 lines) and constructed an event-activity network with about 150,000 vertices and about 480,000 arcs. About 7,000,000 passengers' flows are simulated by finding a Wardrop equilibrium in the event-activity network.

\section{Event-activity network}

Event-activity networks are widely used in the timetable design [11, 12]. Given a timetable $\Pi$, we construct an event-activity network as follows. Let $V$ be the set of stations and $H$ be the set of trains. We also denote by $V_{\text {tran }}$ the set of stations shared by two lines, where passengers can transfer from one line to another. We define

$$
\begin{aligned}
\mathcal{E}_{\text {arr }} & =\{(g, v, \text { arr }) \mid \text { train } g \in H \text { arrives at station } v \in V\}, \\
\mathcal{E}_{\text {dep }} & =\{(g, v, \text { dep }) \mid \text { train } g \in H \text { departs from station } v \in V\} .
\end{aligned}
$$

The sets $\mathcal{E}_{\text {arr }}$ and $\mathcal{E}_{\text {dep }}$ represent arrival events and departure events. Each $i \in \mathcal{E}_{\text {arr }} \cup \mathcal{E}_{\text {dep }}$ has the arrival or departure time in the timetable $\Pi$, denoted by $\Pi_{i}$. Next, we define arc sets

$$
\begin{aligned}
\mathcal{A}_{\text {drive }} & =\{((g, v, \text { dep }),(g, u, \text { arr })) \mid \text { train } g \text { goes directly from } v \text { to } u\}, \\
\mathcal{A}_{\text {wait }} & =\left\{((g, v, \text { arr }),(g, v, \text { dep })) \in \mathcal{E}_{\text {arr }} \times \mathcal{E}_{\text {dep }}\right\}, \\
\mathcal{A}_{\text {tran }} & =\left\{\left((g, v, \text { arr }),\left(g^{\prime}, v, \text { dep }\right)\right) \mid v \in V_{\text {tran }}, \Pi_{\left(g^{\prime}, v, \text { dep }\right)}\right. \text { is the earliest departure time } \\
& \text { satisfying } \left.\Pi_{(g, v, \text { arr })}+L_{\text {tran }} \leq \Pi_{\left(g^{\prime}, v, \text { dep }\right)}\right\},
\end{aligned}
$$




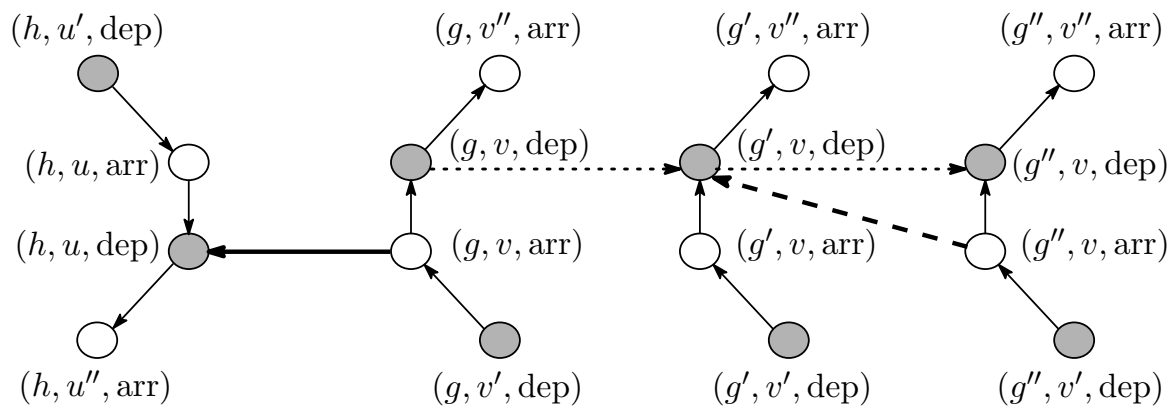

Figure 1 An event-activity network, where the solid lines denote $\operatorname{arcs}$ of $\mathcal{A}_{\text {drive }}$ and $\mathcal{A}_{\text {wait }}$, the dotted lines correspond to arcs of $\mathcal{A}_{\text {next }}$, the bold lines represent arcs of $\mathcal{A}_{\text {tran }}$, and the dashed lines indicate arcs of $\mathcal{A}_{\text {change. }}$ White and gray vertices represent arrival and departure events, respectively.

where $L_{\text {tran }}$ denotes the time needed for transfer. The sets $\mathcal{A}_{\text {drive }}, \mathcal{A}_{\text {wait }}$, and $\mathcal{A}_{\text {tran }}$ represent driving activities, waiting activities, and transfer activities, respectively. We also define

$$
\begin{aligned}
\mathcal{A}_{\text {change }} & =\left\{\left((g, v, \text { arr }),\left(g^{\prime}, v, \text { dep }\right)\right) \mid \Pi_{(g, v, \text { arr })}<\Pi_{\left(g^{\prime}, v, \text { arr }\right)}, \Pi_{\left(g^{\prime}, v, \text { dep }\right)}<\Pi_{(g, v, \text { dep })}\right\}, \\
\mathcal{A}_{\text {next }} & =\left\{\left((g, v, \text { dep }),\left(g^{\prime}, v, \text { dep }\right)\right) \mid g^{\prime} \text { is the next train to } g\right\} .
\end{aligned}
$$

The set $\mathcal{A}_{\text {tran }}$ expresses transfers between different lines, while $\mathcal{A}_{\text {change }}$ deals with transfers between different types of trains in the same line.

In the event-activity network, the set of vertices and the set of arcs are given by

$$
\mathcal{E}=\mathcal{E}_{\text {arr }} \cup \mathcal{E}_{\text {dep }}, \quad \mathcal{A}=\mathcal{A}_{\text {drive }} \cup \mathcal{A}_{\text {wait }} \cup \mathcal{A}_{\text {tran }} \cup \mathcal{A}_{\text {change }} \cup \mathcal{A}_{\text {next }} .
$$

Figure 1 depicts an example of an event-activity network.

We explain the usefulness of $\mathcal{A}_{\text {next }}$ and $\mathcal{A}_{\text {change }}$ in Figure 1. Suppose that a passenger waits for train $g$ at $v$. If train $g$ is very crowded, he or she has choices to get on $g^{\prime}$ or $g^{\prime \prime}$ instead of $g$. The corresponding route is expressed by a path with arcs of $\mathcal{A}_{\text {next }}$.

Next, let $g^{\prime}$ be an express train and $g^{\prime \prime}$ be a local train. Suppose that $g^{\prime}$ arrives at $v$ after $g^{\prime \prime}$ arrives and departs from $v$ before $g^{\prime \prime}$ departs. In this situation, we have four kinds of passengers moving along

- $\left(\left(g^{\prime}, v\right.\right.$, arr $),\left(g^{\prime}, v\right.$, dep $\left.)\right) \in \mathcal{A}_{\text {wait }}$ : Passengers use only express train $g^{\prime}$.

- $\left(\left(g^{\prime \prime}, v\right.\right.$, arr $),\left(g^{\prime \prime}, v\right.$, dep $\left.)\right) \in \mathcal{A}_{\text {wait }}:$ Passengers use only local train $g^{\prime \prime}$.

- $\left(\left(g^{\prime \prime}, v\right.\right.$, arr $),\left(g^{\prime}, v\right.$, dep $\left.)\right) \in \mathcal{A}_{\text {change }}:$ Passengers transfer from $g^{\prime \prime}$ to $g^{\prime}$.

- $\left(\left(g^{\prime}, v\right.\right.$, arr $),\left(g^{\prime}, v\right.$, dep $\left.)\right) \in \mathcal{A}_{\text {wait }}$ and $\left(\left(g^{\prime}, v\right.\right.$, dep $),\left(g^{\prime \prime}, v\right.$, dep $\left.)\right) \in \mathcal{A}_{\text {next }}:$ Passengers transfer from $g^{\prime}$ to $g^{\prime \prime}$.

The combination of $\mathcal{A}_{\text {change }}$ and $\mathcal{A}_{\text {next }}$ enables to express them faithfully. In a Wardrop equilibrium, some passengers do not select the shortest route to avoid crowded trains. We can express all kinds of passengers by using the event-activity network defined above.

\section{Analysis of congestion rate in event-activity network}

We focus on five railway lines in Tokyo: Keio Line, Keio Takao Line, Keio Sagamihara Line, Keio Inokashira Line, and Toei Shinjuku Line ${ }^{1}$. We collectively call these five lines Keio Railway Lines. Figure 2 shows the railway map of Keio Railway Lines.

1 The first four lines are operated by the private railway operator Keio Corporation, and the last line is operated by Bureau of Transportation, Tokyo Metropolitan Government. 


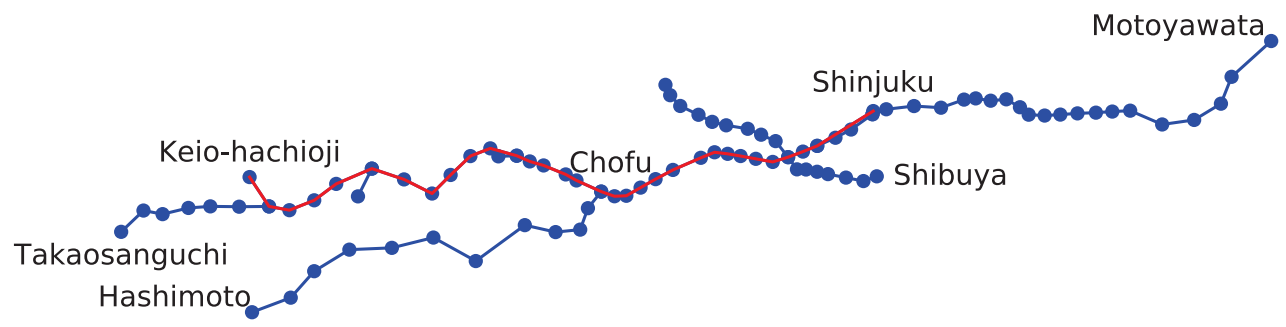

Figure 2 Keio Railway Map, where the red line denotes Keio Line (Keio-hachioji-Shinjuku) and the blue lines represent Keio Takao Line, Keio Sagamihara Line, Keio Inokashira Line, and Toei Shinjuku Line.

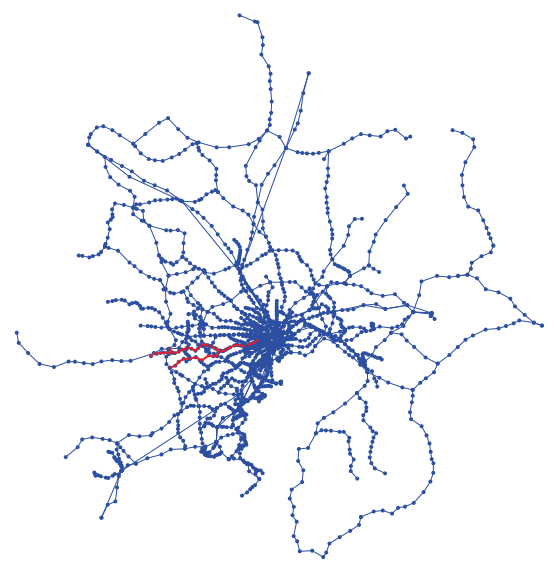

Figure 3 Tokyo Railway network with 2128 nodes and 3041 edges, where red edges represent Keio Railway Lines.

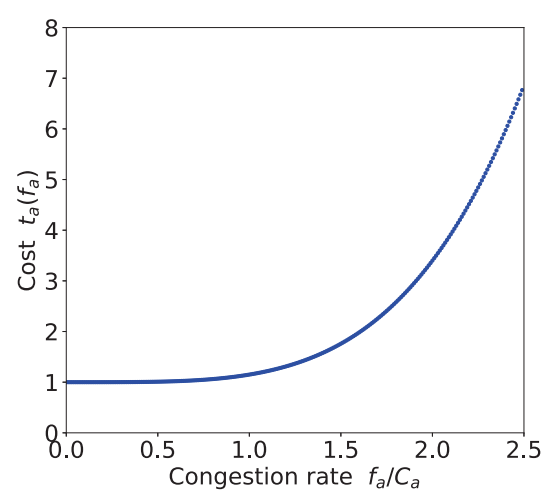

Figure 4 BPR function with $\alpha=0.15$ and $\beta=4$.

We compute congestion rates of each train during morning rush hour from 4:30 to 9:30 for 2016 timetable. In order to find a Wardrop equilibrium, we need to estimate OD pairs with available data. We first construct a railway network in the Tokyo metropolitan area, which is given in Figure 3. Next we compute the optimal route for each pair of stations with respect to distance and the number of transfers, and then extract routes which use Keio Railway Lines from them. The number of obtained OD pairs is 418,394, which are divided into four types:

- use only Keio Railway Lines.

- first use another line and then transfer to Keio Railway Lines.

- first use Keio Railway Lines and then transfer to another line.

- use another line, transfer to Keio Railway Lines, and transfer to another line again.

We now combine the extracted routes with commuter passengers' data in the report [10] published by Ministry of Land, Infrastructure, Transport and Tourism. This report lists 83,838 OD pairs, and each OD pair has the following information: origin station, destination station, and the number of passengers. Note that each OD pair does not have information about departure time. By deleting OD pairs which are not listed in the passengers' data from the extracted 418,394 OD pairs, we obtain 9,712 OD pairs and 805,053 passengers who get on Keio Railway Lines.

Let us summarize the obtained data. For each OD pair, we know the origin/destination station (not necessarily in Keio Railway Lines), the first/last station in Keio Railway Lines, 


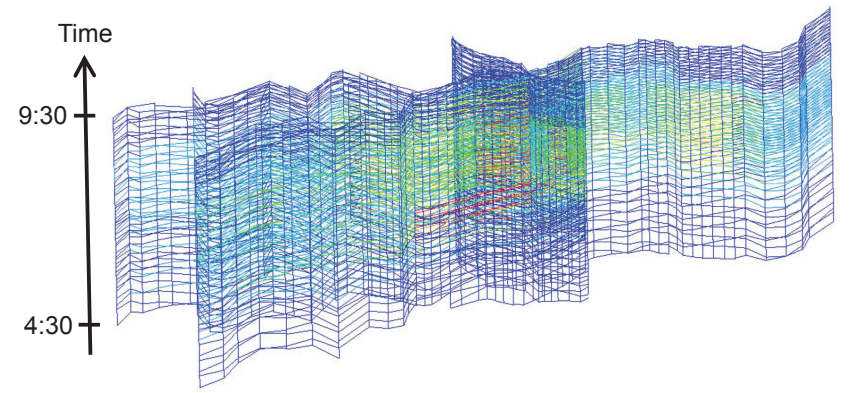

Figure 5 Computational results for a Wardrop equilibrium in the event-activity network of 2016 timetable. Red, green, and blue arcs represent trains with congestion rate more than $200 \%$, around $150 \%$, and less than $100 \%$, respectively.

and the number of passengers. Based on these data, we determine when and which train each passenger boards by finding a Wardrop equilibrium in the event-activity network.

In order to assign passengers to the event-activity network, we add the following vertices and arcs. Let $\mathcal{C}$ be the set of 9,712 OD pairs. For an OD pair $k \in \mathcal{C}$, we denote by $v_{\text {org }}(k)$ the first station in Keio Railway Lines along the route. Let us define

$$
\mathcal{E}_{\text {org }}=\{k \mid k \in \mathcal{C}\}, \quad \mathcal{A}_{\text {org }}=\left\{\left(k,\left(g, v_{\text {org }}(k), \text { dep }\right)\right) \mid t_{k}^{0} \leq \Pi_{\left(g, v_{\text {org }}(k), \text { dep }\right)} \leq t_{k}^{1}\right\},
$$

where $t_{k}^{0}$ and $t_{k}^{1}$ are determined by distance between $v_{\text {org }}(k)$ and the final destination station.

In computation of a Wardrop equilibrium in the event-activity network $(\mathcal{E}, \mathcal{A})$, we use the BPR function (Bureau of Public Roads [2]) as a cost function:

$$
\begin{aligned}
& t_{a}\left(f_{a}\right)=L_{a}\left(1+\alpha\left(\frac{f_{a}}{C_{a}}\right)^{\beta}\right) \quad\left(a \in \mathcal{A}_{\text {drive }} \cup \mathcal{A}_{\text {wait }}\right), \\
& t_{a}\left(f_{a}\right)=L_{a} \quad\left(a \in \mathcal{A} \backslash\left(\mathcal{A}_{\text {drive }} \cup \mathcal{A}_{\text {wait }}\right)\right),
\end{aligned}
$$

where $f_{a}$ denotes a flow on $\operatorname{arc} a \in \mathcal{A}, L_{a}$ is travel time for $a \in \mathcal{A}$, and $C_{a}$ is the capacity of a train. The term $f_{a} / C_{a}$ represents congestion rate. We set parameters $\alpha$ and $\beta$ by $\alpha=0.15$ and $\beta=4$.

Figure 4 depicts a BPR function with $L_{a}=1, \alpha=0.15$, and $\beta=4$. In a case with $f_{a} / C_{a} \leq 1, t_{a}\left(f_{a}\right)$ is almost the same as $L_{a}$. The value of $t_{a}\left(f_{a}\right)$ suddenly increases with $f_{a} / C_{a}=1.5$ and reaches into $6.8 L_{a}$ when $f_{a} / C_{a}=2.5$. This function describes that passengers do not care for congestion if the congestion rate $f_{a} / C_{a}$ is small but congestion effects have a bigger impact on passengers' behavior in trains with higher congestion rate.

We solve (1) by the Frank-Wolfe method to find a Wardrop equilibrium. Figure 2 depicts the railway network which we focus on in this paper. Figure 5 shows computational results for a Wardrop equilibrium in the event-activity network of 2016 timetable. We can see that trains between 7:00 and 8:30 have especially high congestion rates in the section between Chofu and Shinjuku, which matches the real situation in Keio Railway Lines. Table 2 describes trains with congestion rate more than $220 \%$.

\section{Approximation model for passenger flows in event-activity network}

Given stopping patterns and timetables, we can evaluate them with computational results for a Wardrop equilibrium in the event-activity network. A basic idea to find optimal 
Table 2 Computational results for trains with congestion rates more than $220 \%$.

\begin{tabular}{c|c|c|c}
\hline Train type & Successive stations & Time & Congestion rate \\
\hline Semi-Express & Chitose-kayasuyama $\rightarrow$ Sakurajosui & $7: 10$ & $234.98 \%$ \\
Local & Shimo-takaido $\rightarrow$ Meidaimae & $8: 24$ & $223.49 \%$ \\
Local & Sasazuka $\rightarrow$ Shinjuku & $7: 09$ & $221.18 \%$ \\
Local & Shimo-takaido $\rightarrow$ Meidaimae & $8: 06$ & $221.16 \%$ \\
Local & Daitabashi $\rightarrow$ Sasazuka & $8: 22$ & $221.09 \%$ \\
Express & Sakurajosui $\rightarrow$ Meidaimae & $8: 25$ & $220.13 \%$ \\
\hline
\end{tabular}

Table 3 Comparison of the event-activity network and the train type network.

\begin{tabular}{c|c|c|c|c}
\hline & \#nodes & \#arcs & \#passengers & $\begin{array}{c}\text { Computational time for } \\
\text { a Wardrop equilibrium }\end{array}$ \\
\hline Event-activity network (4:30-9:30) & 15,667 & 24,482 & 805,053 & 2026 [sec.] \\
\hline Train type network (7:00-8:30) & 1,552 & 1,952 & 627,406 & 0.357 [sec.] \\
\hline
\end{tabular}

stopping patterns is to update stopping patterns iteratively based on the obtained Wardrop equilibrium. In this approach, however, we need to find a Wardrop equilibrium many times. As shown in Table 3, computing a Wardrop equilibrium in the event-activity network takes about 34 minutes. In order to reduce computational time, we introduce a small network model such that a Wardrop equilibrium in this network approximates a Wardrop equilibrium in the event-activity network.

We now introduce a train type network. Remember that $V$ denotes the set of stations. Let $T=\{0,1,2,3,4,5\}$ be the set of train types, where each element corresponds to a train type as follows:

0: Local, 1: Rapid, 2: Semi-Express, 3: Express, 4: Semi-Special Express, 5: Special Express.

We denote stopping patterns $\mathcal{S}$ by the set of pairs of a train type and a station where the train makes stops:

$$
\mathcal{S}=\{(v, t) \mid \text { train type } t \in T \text { stops at station } v \in V\} .
$$

Given stopping patterns $\mathcal{S}$, we define

$$
\begin{aligned}
\overline{\mathcal{A}}_{\text {drive }} & =\{((v, t),(u, t)) \in \mathcal{S} \times \mathcal{S} \mid t \text { goes directly from } v \text { to } u\}, \\
\overline{\mathcal{A}}_{\text {change }} & =\{((v, t), v) \in \mathcal{S} \times V\} \cup\{(v,(v, t)) \in V \times \mathcal{S}\} .
\end{aligned}
$$

Arcs of $\overline{\mathcal{A}}_{\text {change }}$ are used when passengers transfer to a different type of train. Here, we distinguish two lines traveling in opposite directions along the same route. Figure 6 shows an example of the train type network $\mathcal{G}(\mathcal{S})=\left(\mathcal{S} \cup V, \overline{\mathcal{A}}_{\text {drive }} \cup \overline{\mathcal{A}}_{\text {change }}\right)$.

We focus on morning rush hour between 7:00 and 8:30 and compute passenger flows with $\mathcal{S}$ determined from 2016 timetable. A cost function in the train type network is defined by

$$
\bar{t}_{a}\left(f_{a}\right)=L_{a}\left(1+\alpha\left(\frac{f_{a}}{N_{a} C_{a}}\right)^{\beta}\right) \quad\left(a \in \overline{\mathcal{A}}_{\text {drive }}\right),
$$

where $N_{a}$ is introduced as the number of trains of the same type driving in the target period. Travel time $L_{a}$ is set to be longer than that for a superior train on the basis of the timetable. 


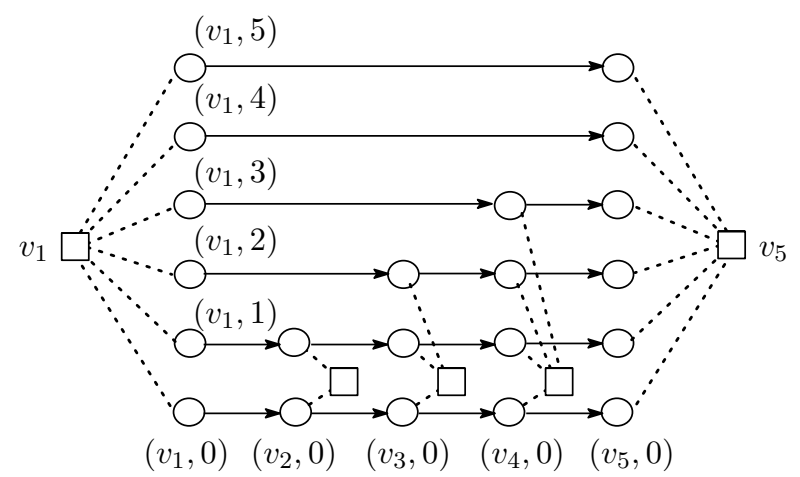

Figure 6 An example of a train type network $\mathcal{G}(\mathcal{S})$, where the dotted lines represent arcs in both directions.

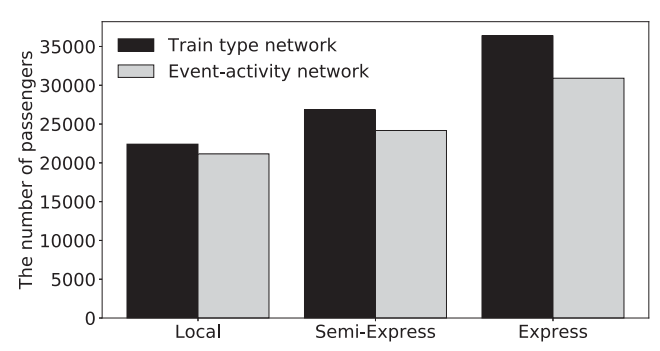

Figure 7 The number of passengers in trains bound for Shinjuku at Chofu.

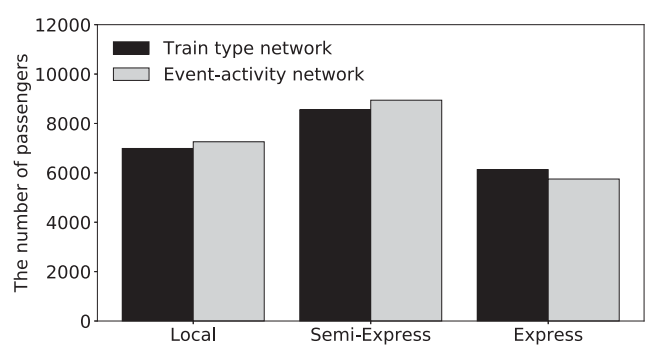

Figure 8 The number of passengers in trains bound for Shinjuku at Hashimoto.

We remark that this function is obtained by adding $N_{a}$ in the denominator to (2). For $a \in \overline{\mathcal{A}}_{\text {change, }}$ we set $\bar{t}_{a}\left(f_{a}\right)$ based on the time needed for transfer.

We make use of OD pairs between 7:00 and 8:30 obtained from the computational results in Section 4. Table 3 compares the train type network and the event-activity network. We can compute a Wardrop equilibrium in the train type network much faster than in the event-activity network.

By finding a Wardrop equilibrium in the train type network, we obtain the number of passengers who get on trains of type $t \in T$ for each pair of successive stations. We now check the validity of passenger flows in the train type network. Figures $7-8$ compare the number of passengers in trains with each type between 7:00 and 8:30 obtained with the train type network to that obtained with the event-activity network. Keio Line has three types of trains bound for Shinjuku (Local, Semi-Express, Express) in the target period. We can see that flows in the train-type network succeed in approximating passenger flows in the event-activity network. This is attributed to the fact that the congestion rate of each train is very high during morning rush hour.

\section{Local search algorithm to optimize stopping patterns}

If we are given stopping patterns $\mathcal{S}$, we evaluate $\mathcal{S}$ by using a Wardrop equilibrium in the train type network $\mathcal{G}(\mathcal{S})$ as follows. Let $f_{a}$ be a passenger flow obtained by computing a 
Wardrop equilibrium in $\mathcal{G}(\mathcal{S})$. A function to evaluate $\mathcal{S}$ is defined by

$$
\operatorname{eval}(\mathcal{S})=\sum_{a \in \overline{\mathcal{A}}_{\text {drive } \cup \overline{\mathcal{A}}_{\text {change }}}} f_{a} \cdot \bar{t}_{a}\left(f_{a}\right)+\sum_{v \in V} c_{v}(\mathcal{S}),
$$

where $c_{v}(\mathcal{S})$ denotes the number of train types except Local which make a stop at $v$.

The first term is determined by congestion rates and travel time. Consider the train type network $\mathcal{G}(\mathcal{S})$ in Figure 6 as an example. The second term $c_{v}(\mathcal{S})$ is given by $\sum_{v \in V} c_{v}(\mathcal{S})=16$, because we have $c_{v_{1}}(\mathcal{S})=5, c_{v_{2}}(\mathcal{S})=1, c_{v_{3}}(\mathcal{S})=2, c_{v_{4}}(\mathcal{S})=3, c_{v_{5}}(\mathcal{S})=5$.

Since the value of the first term is much larger than that of the second term, eval $(\mathcal{S})$ is affected by the first term in most cases. The second term is useful when there exist stopping patterns $\mathcal{S}$ and $\mathcal{S}^{\prime}$ such that the values of the first term in $\operatorname{eval}(\mathcal{S})$ and $\operatorname{eval}\left(\mathcal{S}^{\prime}\right)$ are exactly equal. In this case, we select the stopping patterns with smaller value of the second term, because superior trains are desirable to stop at fewer stations.

For example, consider the train type network in Figure 6 again. Let $\mathcal{S}$ be the stopping patterns given in Figure 6. Assume that we have stopping patterns $\mathcal{S}_{1}=\mathcal{S} \cup\left\{\left(v_{3}, 3\right)\right\}$ and $\mathcal{S}_{2}=\mathcal{S} \cup\left\{\left(v_{3}, 3\right),\left(v_{3}, 4\right)\right\}$ such that the values of the first term are equal. Then $\operatorname{eval}\left(\mathcal{S}_{1}\right)<\operatorname{eval}\left(\mathcal{S}_{2}\right)$ holds. In the stopping patterns $\mathcal{S}_{1}$, Express makes a stop at $v_{3}$ but Semi-Special Express does not, while both Express and Semi-Special Express make a stop at $v_{3}$ in $\mathcal{S}_{2}$. The inequality $\operatorname{eval}\left(\mathcal{S}_{1}\right)<\operatorname{eval}\left(\mathcal{S}_{2}\right)$ with the same values of the first term means that adding only $\left(v_{3}, 3\right)$ is enough to reduce the first term and Semi-Special Express does not need to stop at $v_{3}$.

Our problem is to find $\mathcal{S}$ which minimizes eval $(\mathcal{S})$. We find $\mathcal{S}$ by the following local search algorithm. Let $\mathcal{S}$ be the current solution. We repeat replacing $\mathcal{S}$ with a better solution in its neighborhood. If we cannot find a better solution, the algorithm outputs $\mathcal{S}$ and terminates.

In designing local search algorithms, an initial solution, a function to evaluate solutions, a move strategy, and a neighborhood are important ingredients. An initial solution $\mathcal{S}_{0}$ is given by $\mathcal{S}_{0}=\left\{(v, t) \mid v \in V_{\text {leaf }}, t \in T\right\} \cup\{(v, 0) \mid v \in V\}$, where $V_{\text {leaf }}$ is the set of Shinjuku and stations corresponding to leaves (vertices with degree one) of the network given in Figure 2. The set $\mathcal{S}_{0}$ means that every train stops at stations in $V_{\text {leaf }}$ and local trains $(t=0)$ stop at all stations. We use (6) as an evaluation function. In addition, we adopt the first admissible move strategy, i.e., when we find a better solution in its neighborhood, we move to the solution immediately. A more sophisticated move strategy is presented at the end of this section.

For the current solution $\mathcal{S}$, we use the following two kinds of neighborhoods, called $N_{\text {op }}(\mathcal{S})$ and $N_{\mathrm{cl}}(\mathcal{S})$. Let $\left(v, t^{\prime}\right) \notin \mathcal{S}$. An opening operation is an operation which adds $\left\{(v, t) \mid t \leq t^{\prime}\right\}$ to $\mathcal{S}$. The neighborhood $N_{\text {op }}(\mathcal{S})$ is defined as the set of all solutions which can be obtained from $\mathcal{S}$ by an opening operation. For $\left(v, t^{\prime}\right) \in \mathcal{S} \backslash \mathcal{S}_{0}$, we use a closing operation, which deletes $\left\{(v, t) \mid t \geq t^{\prime}\right\}$ from $\mathcal{S}$. The neighborhood $N_{\mathrm{cl}}(\mathcal{S})$ is defined as the set obtained by a closing operation. We remark that these operations are defined so that if a train of type $t$ stops at $v$, then trains of inferior types also stop at $v$.

Since $\mathcal{S}_{0}$ is set to be an initial solution, we design an algorithm which emphasizes an opening operation over a closing operation. If we cannot find a better solution in $N_{\text {op }}(\mathcal{S})$, we exploit a closing operation. The outline of our algorithm is as follows. In the algorithm, $c$ denotes the number of fails to find a better solution than the current solution $\mathcal{S}$. We set a parameter $\gamma$ by $\gamma=50$. 
Table 4 Comparison of four move strategies.

\begin{tabular}{c|c|c|c|c|c|c}
\hline & \multicolumn{2}{|c|}{ Proposed } & \multicolumn{2}{c|}{ FA } & BA1 & BA2 \\
\hline & Best & Average & Best & Average & BA1 & BA2 \\
\hline Eval. value & $16,056,810$ & $16,205,030$ & $16,084,948$ & $16,219,808$ & $16,144,254$ & $16,147,615$ \\
Time (sec.) & 598 & 332 & 296 & 454 & 443 & 651 \\
\#iteration & 88 & 55 & 64 & 75 & 15 & 21 \\
\hline
\end{tabular}

\section{Local search algorithm with first admissible move strategy}

Step 0. Set $\mathcal{S} \leftarrow \mathcal{S}_{0}$ and $c \leftarrow 0$.

Step 1. Choose $\left(v, t^{\prime}\right) \notin \mathcal{S}$ randomly.

Step 2. Let $\mathcal{S}^{\prime}$ denote the set obtained by an opening operation with $\left(v, t^{\prime}\right)$. If eval $\left(\mathcal{S}^{\prime}\right)<$ $\operatorname{eval}(\mathcal{S})$, then set $\mathcal{S} \leftarrow \mathcal{S}^{\prime}, c \leftarrow 0$ and return to Step 1.

Step 3. Set $c \leftarrow c+1$. If $c<\gamma$, then return to Step 1. Otherwise set $c \leftarrow 0$.

Step 4. Choose $\left(v, t^{\prime}\right) \in \mathcal{S} \backslash \mathcal{S}_{0}$ randomly.

Step 5. Let $\mathcal{S}^{\prime}$ denote the set obtained by a closing operation with $\left(v, t^{\prime}\right)$. If eval $\left(\mathcal{S}^{\prime}\right)<$ $\operatorname{eval}(\mathcal{S})$, then set $\mathcal{S} \leftarrow \mathcal{S}^{\prime}, c \leftarrow 0$ and return to Step 1 .

Step 6. Set $c \leftarrow c+1$. If $c<\gamma$, then return to Step 4. Otherwise output $\mathcal{S}$ and stop.

We adopt $N_{\mathrm{op}}(\mathcal{S})$ as a neighborhood in Steps 1-2 and $N_{\mathrm{cl}}(\mathcal{S})$ in Steps $4-5$. We try to find a better solution by an opening operation while $c<\gamma$, but switch to a closing operation if we fail $\gamma$ times. The algorithm terminates if we fail $\gamma$ times by an opening operation and $\gamma$ times by a closing operation. It should be noted that we need to compute a Wardrop equilibrium in the train type network $\mathcal{G}\left(\mathcal{S}^{\prime}\right)$ in Steps 2 and 5.

The first admissible move strategy has a drawback that it sometimes selects bad moves, because we choose $\left(v, t^{\prime}\right)$ randomly in Steps 1 and 4 . With an aim for a better performance of the first admissible move strategy, we present a new strategy, where we find several better solutions than the current solution and then move to the best solution among them.

Let $d$ denote the number of better solutions which we find before moving to the next solution. We set a parameter $\kappa$ by $\kappa=5$ (the digit in the tens place of $\gamma$ ). A detail of the strategy in Steps $1-3$ is described below:

\section{Sophisticated move strategy}

- At the first of the algorithm, we set $d \leftarrow \kappa$.

- If $d<\kappa$ and we execute Step 1 at most $\kappa$ times to find $d$ better solutions, update $d \leftarrow d+1$.

- If we execute Step 1 more than $\kappa$ times to find $d$ better solutions, we decrease the value of $d$. In the case study given in Section 7 , we update $d \leftarrow d-q$, where $q$ is the digit in the tens place of the number of executions of Step 1.

The number of solutions to be checked depends on iterations. If the neighborhood has a lot of better solutions, we select where to move after checking several solutions. If it is not easy to find a better solution, the strategy adopts the first admissible move strategy $(d=1)$. A similar strategy is also used in Steps 4-6. 

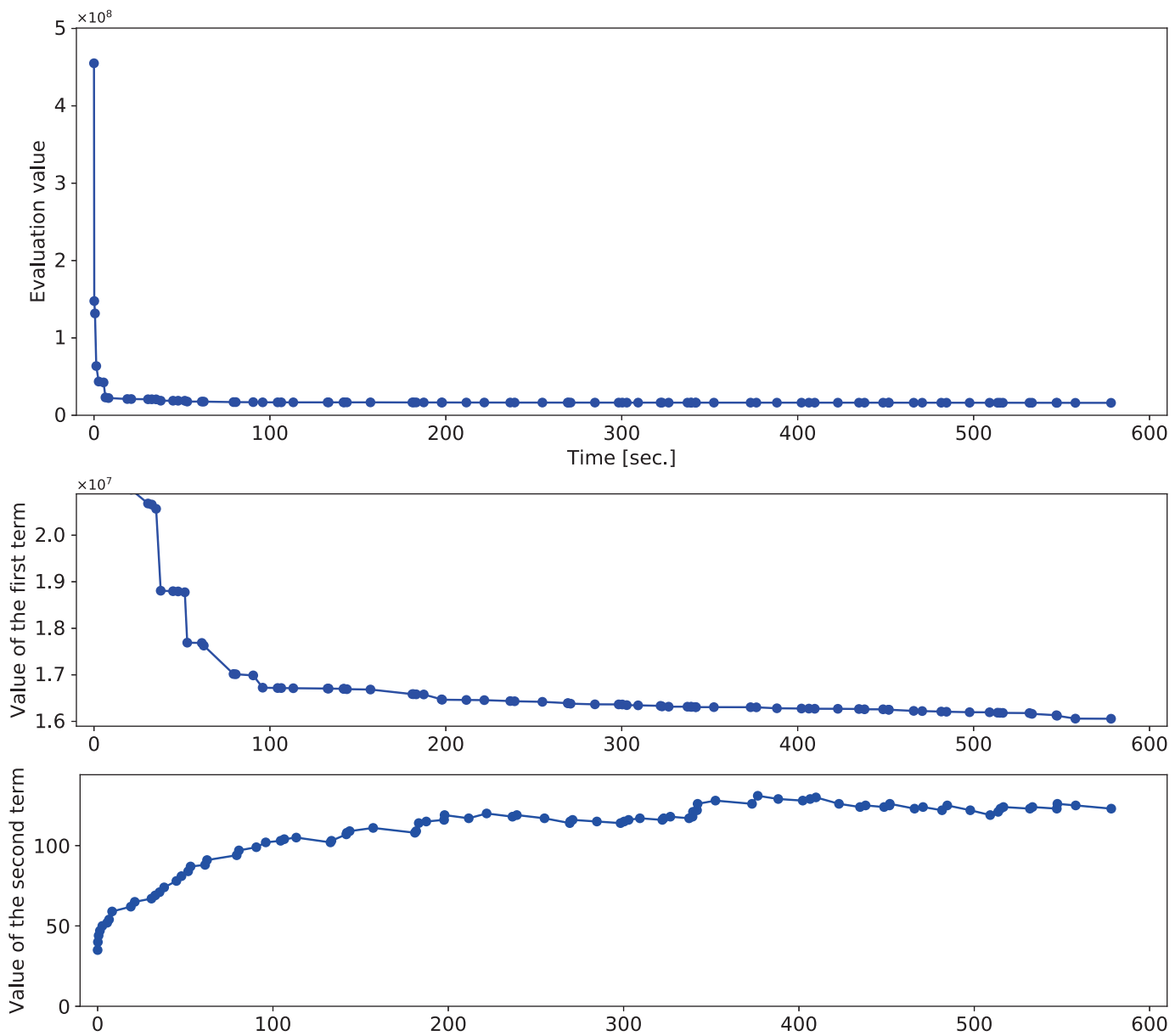

Figure 9 Evaluation value eval $(\mathcal{S})$ (top), value of the first term in eval $(\mathcal{S})$ (middle), and value of the second term in $\operatorname{eval}(\mathcal{S})$ (bottom), where $\bullet$ represents when we move to a better solution.

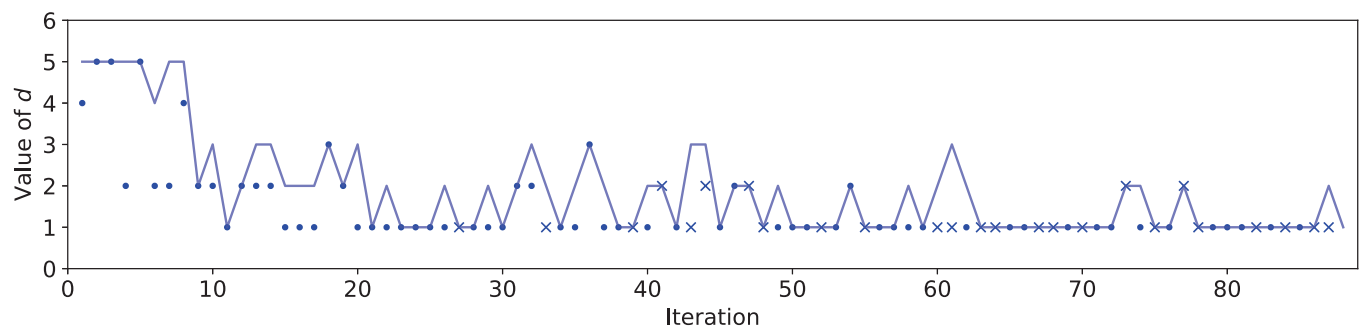

Figure 10 Value of $d$ (solid line) and adopted operation in each iteration, where $\bullet$ and $\mathrm{x}$ represent an opening operation and a closing operation, respectively. The height of $\bullet$ and $\mathrm{x}$ shows when we find the corresponding solution in $d$ trials.

\section{Case study: Keio Railway Lines}

We apply the local search algorithm described in Section 6 to Keio Railway Lines in the time period from 7:00 to 8:30. Table 4 compares solutions obtained by the following four move strategies: sophisticated move strategy (Proposed), first admissible move strategy (FA), best 


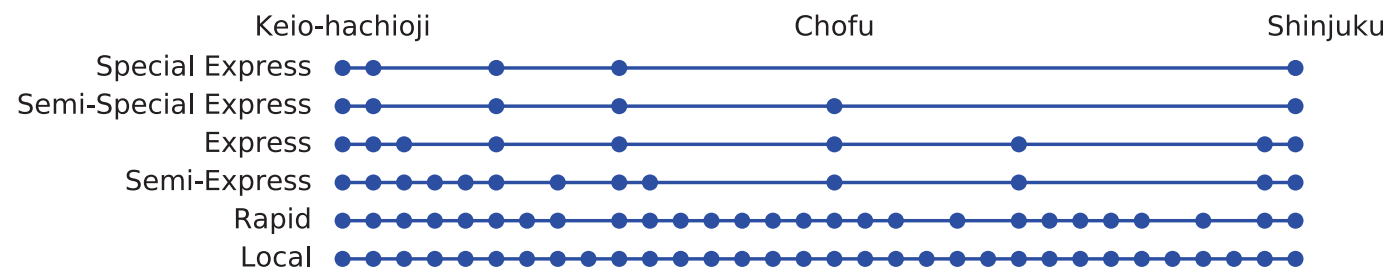

Figure 11 Stopping patterns on Keio Line obtained by the local search algorithm.

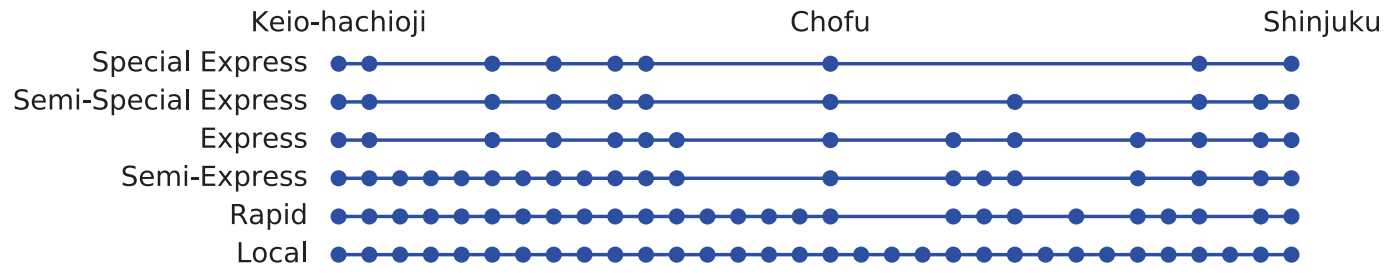

Figure 12 Stopping patterns on Keio Line in 2016.

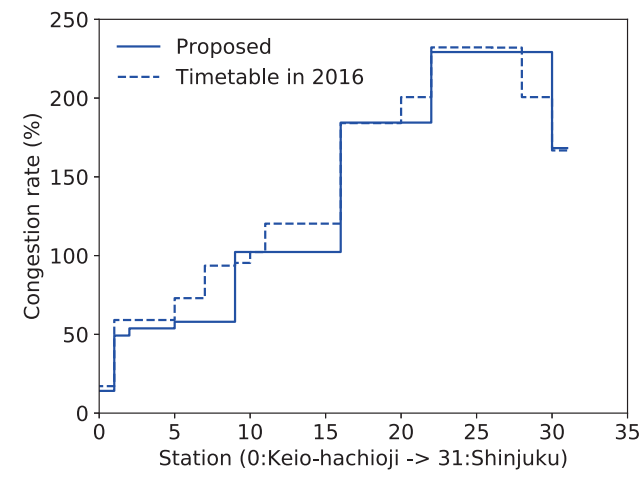

Figure 13 Comparison of congestion rates for Express $(t=3)$.

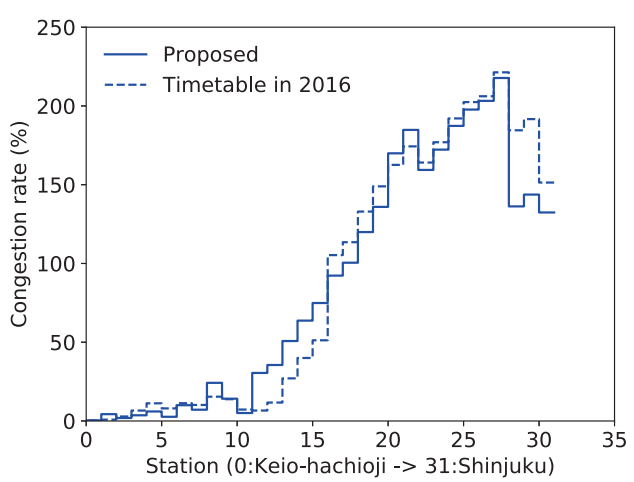

Figure 15 Comparison of congestion rates for Local $(t=0)$.

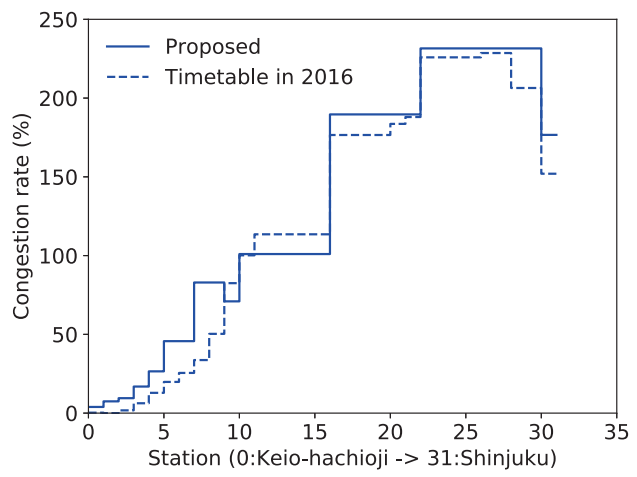

Figure 14 Comparison of congestion rates for Semi-Express $(t=2)$.

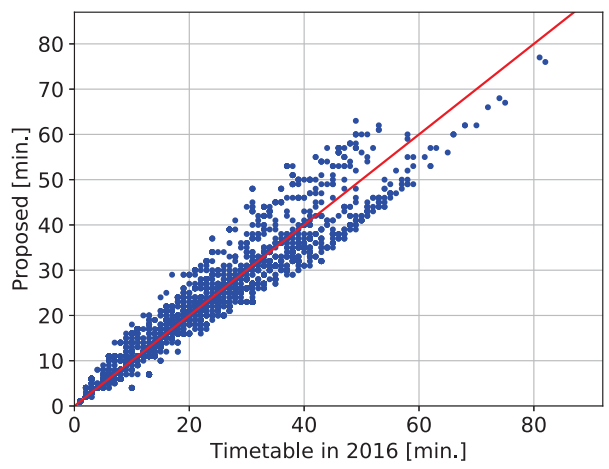

Figure $\mathbf{1 6}$ Comparison of travel time, where a red line indicates that travel time is the same. 
Table 5 Top ten arcs of $\overline{\mathcal{A}}_{\text {drive }}$ for the obtained stopping patterns.

\begin{tabular}{c|c|c}
\hline Train type & Successive stations & Congestion rate \\
\hline Semi-Express & Chitose-kayasuyama $\rightarrow$ Sasazuka & $231.54 \%$ \\
Express & Chitose-kayasuyama $\rightarrow$ Sasazuka & $229.22 \%$ \\
Local & Shimo-takaido $\rightarrow$ Meidaimae & $217.76 \%$ \\
Local & Sakurajosui $\rightarrow$ Shimo-takaido & $203.23 \%$ \\
Local & Kami-kitazawa $\rightarrow$ Sakurajosui & $197.71 \%$ \\
Semi-Express & Chofu $\rightarrow$ Chitose-kayasuyama & $189.63 \%$ \\
Local & Hachiman-yama $\rightarrow$ Kami-kitazawa & $187.33 \%$ \\
Local & Sengawa $\rightarrow$ Chitose-kayasuyama & $184.79 \%$ \\
Express & Chofu $\rightarrow$ Chitose-kayasuyama & $184.44 \%$ \\
Semi-Express & Sasazuka $\rightarrow$ Shinjuku & $176.63 \%$ \\
\hline
\end{tabular}

Table 6 Top ten arcs of $\overline{\mathcal{A}}_{\text {drive }}$ for 2016 timetable.

\begin{tabular}{c|c|c}
\hline Train type & Successive stations & Congestion rate \\
\hline Express & Chitose-kayasuyama $\rightarrow$ Sakurajosui & $232.18 \%$ \\
Express & Sakurajosui $\rightarrow$ Meidaimae & $232.04 \%$ \\
Semi-Express & Sakurajosui $\rightarrow$ Meidaimae & $228.60 \%$ \\
Semi-Express & Chitose-kayasuyama $\rightarrow$ Sakurajosui & $225.86 \%$ \\
Local & Shimo-takaido $\rightarrow$ Meidaimae & $221.34 \%$ \\
Semi-Express & Meidaimae $\rightarrow$ Sasazuka & $206.40 \%$ \\
Local & Sakurajosui $\rightarrow$ Shimo-takaido & $206.15 \%$ \\
Local & Kami-kitazawa $\rightarrow$ Sakurajosui & $202.46 \%$ \\
Express & Meidaimae $\rightarrow$ Sasazuka & $200.61 \%$ \\
Express & Tsutsujigaoka $\rightarrow$ Chitose-kayasuyama & $200.61 \%$ \\
\hline
\end{tabular}

admissible move strategy (BA1), and modified best admissible move strategy (BA2). For the first two strategies, we run the algorithm 50 times. In BA1, we move to the best solution among all the solution obtained by an opening operation and a closing operation, while BA2 adopts a closing operation only when we cannot obtain a better solution by using an opening operation. We can see that all the evaluation values in Table 4 greatly improve 17,565, 000, which is the evaluation value for the stopping patterns of 2016 timetable.

BA2 uses smaller neighborhood than BA1, but leads to longer computational time. This indicates that the number of iterations has a stronger effect on computational time than the neighborhood size. The proposed strategy attains shorter computational time than BA1 and BA2 on average and sometimes finds better solutions.

Next, we analyze the best solution obtained by the proposed strategy, which has the minimum evaluation value in Table 4. Figure 9 depicts a behavior of the evaluation value and Figure 10 shows which operation is adopted in each iteration. In most iterations of the early stage, the algorithm adopts opening operations and $d$ is large. In the latter stage, the algorithm adopts both opening/closing operations and $d=1$ (the first admissible move strategy is selected) in many iterations.

We now focus on Keio Line and further analyze the best solution obtained by the proposed strategy. Keio Line has three types of trains bound for Shinjuku (Local, SemiExpress, Express) and six types of trains bound for Keio-hachioji between 7:00 and 8:30. Figure 11 shows the obtained stopping patterns on Keio Line. Superior trains (Express, Semi- 
Special Express, Special Express) have fewer stops than 2016 timetable given in Figure 12. The obtained stopping patterns emphasize a difference between superior trains and the other trains, while they have common stops with 2016 timetable.

Trains bound for Shinjuku are extremely crowded during morning rush hour. Let us compare congestion rates in Figure 13-15. Figure 13 shows that in the obtained stopping patterns, the maximum congestion rate is almost the same, while passengers who depart from Keio-hachioji suffer less congestion than 2016 timetable. Thus, we conclude that we can make full use of express trains even if we reduce stopping patterns of 2016 timetable. In Figures 14-15, the congestion rate becomes large at stations indexed as 0-10 in Semi-Express and 11-15 in Local. However, this causes no problem because the rate is less than $100 \%$. Moreover, the congestion rate of local trains at stations indexed as $16-20$ and $23-27$ is a bit improved.

Figure 16 compares travel time for each OD pair in the train type network. We can see that a lot of OD pairs whose travel time are more than 60 minutes have shorter travel time, while some passengers with short distance have longer travel time.

Tables 5 and 6 show the top ten arcs of $\overline{\mathcal{A}}_{\text {drive }}$ in the train type network for the obtained stopping patterns and 2016 timetable. We have only 4 arcs with congestion rate more than $200 \%$ in Table 5, while we have 10 arcs in Table 6 . Although the maximum congestion rate is almost the same, the obtained stopping patterns have fewer arcs with congestion rate more than $200 \%$ than 2016 timetable.

\section{Conclusion}

We have presented a local search algorithm to optimize stopping patterns with the evaluation function determined by a Wardrop equilibrium. First, we have computed time-dependent passenger flows subject to congestion phenomena by finding a Wardrop equilibrium in the event-activity network. Then, we have introduced a simple network such that a Wardrop equilibrium in this network approximates a Wardrop equilibrium in the event-activity network, which enables us to devise an efficient algorithm. In a case study for Keio Railway Lines in Tokyo, we have succeeded in relaxing congestion with a small effect on travel time.

It is left for future work to design timetables for the obtained stopping patterns. Another future work is applying the framework of our algorithm to optimize the number of train types and the number of trains of each type, which are determined based on 2016 timetable in this paper.

\section{References}

1 M. Beckmann, C. B. McGuire, and C. B. Winsten. Studies in the Economics of Transportation. Yale University Press, New Haven, 1956.

2 Bureau of Public Roads, U.S. Dept. of Commerce, Urban Planning Division, Washington D.C. Traffic assignment manual, 1964.

3 Z. Cao, Z. Yuan, and S. Zhang. Performance analysis of stop-skipping scheduling plans in rail transit under time-dependent demand. International Journal of Environmental Research and Public Health, 13:707: 1-23, 2016.

4 J. J. Cochran, L. A. Cox, Jr., P. Keskinocak, J.P. Kharoufeh, and J. C. Smith, editors. Wiley Encyclopedia of Operations Research and Management Science. John Wiley \& Sons, Inc., 2011.

5 M. Florian. Untangling traffic congestion: application of network equilibrium models in transportation planning. OR/MS Today, 26:52-57, 1999. 
6 M. Frank and P. Wolfe. An algorithm for quadratic programming. Naval Research Logistics, 3:95-110, 1956.

7 C. Liebchen and R. Möhring. The modeling power of the periodic event scheduling problem: railway timetables and beyond. In Algorithmic methods for railway optimization, volume 4359 of Lecture notes in computer science, pages 3-40, Berlin, 2007. Springer.

8 D.-Y. Lin and Y.-H. Ku. Using genetic algorithms to optimize stopping patterns for passenger rail transportation. Computer-Aided Civil and Infrastructure Engineering, 29:264-278, 2014.

9 Transport Ministry of Land, Infrastructure and Tourism. (In Japanese). URL: http: //www.mlit.go.jp/common/001099730.pdf.

10 Transport Ministry of Land, Infrastructure and Tourism. (In Japanese). URL: http: //www.mlit.go.jp/sogoseisaku/transport/sosei_transport_tk_000035.html.

11 M. E. Schmidt. Integrating Routing Decisions in Public Transportation Problems. Springer, New York, 2014.

12 A. Schöbel. Optimization in Public Transportation. Springer, New York, 2006.

13 A. Schöbel. Line planning in public transportation: models and methods. OR spectrum, 34:491-510, 2012.

14 A. Taguchi. Time dependent traffic assignment model for commuter traffic in Tokyo Metropolitan railway network (in Japanese). Transactions of the Operations Research Society of Japan, 48:85-108, 2005.

15 S. Toriumi, Y. Nakamura, and A. Taguchi. A computation model for delay of commuter train (in Japanese). Communications of the Operations Research Society of Japan, 50:409416, 2005.

16 L. Wang, L. M. Jia, Y. Qin, J. Xu, and W. Mo. A two-layer optimization model for highspeed railway line planning. Journal of Zhejiang University - SCIENCE A, 12:902-912, 2011.

17 Y. Yue, S. Wang, L. Zhou, L. Tong, and M. R. Saat. Optimizing train stopping patterns and schedules for high-speed passenger rail corridors. Transportation Research Part C, 63:126-146, 2016. 